\title{
ARTICLE OPEN miR-140-3p enhanced the osteo/odontogenic differentiation of DPSCs via inhibiting KMT5B under hypoxia condition
}

\author{
Han Zheng ${ }^{1}$, Ning Wang ${ }^{1}$, Le $\mathrm{Li}^{2}$, Lihua Ge ${ }^{1}$, Haichao $\mathrm{Jia}^{3 凶}$ and Zhipeng Fan iD $^{1,4 凶}$
}

\begin{abstract}
Human dental pulp stem cells (DPSCs) have emerged as an important source of stem cells in the tissue engineering, and hypoxia will change various innate characteristics of DPSCs and then affect dental tissue regeneration. Nevertheless, little is known about the complicated molecular mechanisms. In this study, we aimed to investigate the influence and mechanism of miR-140-3p on DPSCs under hypoxia condition. Hypoxia was induced in DPSCs by Cobalt chloride $\left(\mathrm{CoCl}_{2}\right)$ treatment. The osteo/dentinogenic differentiation capacity of DPSCs was assessed by alkaline phosphatase (ALP) activity, Alizarin Red S staining and main osteo/ dentinogenic markers. A luciferase reporter gene assay was performed to verify the downstream target gene of miR-140-3p. This research exhibited that miR-140-3p promoted osteo/dentinogenic differentiation of DPSCs under normoxia environment. Furthermore, miR-140-3p rescued the $\mathrm{CoCl}_{2}$-induced decreased osteo/odontogenic differentiation potentials in DPSCs. Besides, we investigated that miR-140-3p directly targeted lysine methyltransferase 5B (KMT5B). Surprisingly, we found inhibition of KMT5B obviously enhanced osteo/dentinogenic differentiation of DPSCs both under normoxia and hypoxia conditions. In conclusion, our study revealed the role and mechanism of miR-140-3p for regulating osteo/dentinogenic differentiation of DPSCs under hypoxia, and discovered that miR-140-3p and KMT5B might be important targets for DPSC-mediated tooth or bone tissue regeneration.
\end{abstract}

International Journal of Oral Science (2021)13:41

https://doi.org/10.1038/s41368-021-00148-y

\section{INTRODUCTION}

Pulpitis and periapical periodontitis are always irreversible, and nowadays the main therapy is root canal treatment clinically, which has reported with high success rates. ${ }^{1}$ Except for a small part of complications of treatment, the teeth will always be more susceptible to fracture after root canal treatment resulting in a higher incidence of extraction. Mesenchymal stem cells (MSCs)based tissue regeneration has shown great promise in modern medicine. $^{2-4}$ MSCs from different sources have been reported and possessed special characteristics, like self-renewal and differentiation capacities, which have showed great potential for regenerating tooth and maxillofacial tissue regeneration. ${ }^{5}$ Today, the therapeutic effect of MSC-based tissue regeneration is not always satisfactory in diseased microenvironment. ${ }^{6}$ The culture of MSCs is always under normoxia in vitro. Upon administration, MSCs enter the diseased microenvironment of patients and react to the new cues which are actually inflammation and hypoxia, common to most oral disease. The hypoxia microenvironment has been uncovered to exert great influence on the MSCs functions and effects of tissue regeneration. ${ }^{7}$ Researchers have exhibited the different results about whether hypoxia inhibits the osteo/ odontogenic differentiation of dental MSCs. It was reported that characteristics of dental MSCs changed and mineralization decreased under hypoxia. ${ }^{8-10}$ While other studies showed the totally opposite results in that hypoxia upregulated osteo/ odontogenic-related genes in different kinds of MSCs derived from dental tissues. ${ }^{11,12}$ Altogether, the mechanism is still unclear and enhancing MSCs function under hypoxia environments is a key issue for dental tissue regeneration.

The extracellular environment such as a hypoxic microenvironment and intracellular changes like the expression levels of certain microRNAs (miRNAs) could additionally balance and determine MSC function and fate. ${ }^{13}$ MiRNAs, a sort of noncoding RNA molecules, can normally bind to the $3^{\prime}$-untranslated region of their target genes and then take part in the regulation of significant biological processes such as differentiation, survival, and apoptosis. ${ }^{14,15}$ Growing evidence suggested that under hypoxia the expression levels of some miRNAs changed. $\mathrm{CoCl}_{2}$-induced hypoxia upregulated the expression of hypoxia-inducible factor1 (HIF-1) and miR-210 in bone marrow mesenchymal stem cells (BMSCs), which functioned in cell survival under hypoxia. ${ }^{16}$ MiR206, a critical regulator, was decreased in BMSCs under hypoxia, which demonstrated a novel aspect of prosurvival signaling in a hypoxic microenvironment $\left(1 \% \mathrm{O}_{2}\right) .{ }^{17}$ Human dental pulp stem cells (DPSCs) are seen as one of candidate stem cells for applications. ${ }^{18-20}$ In our previous study, we investigated the molecular mechanisms of DPSCs under hypoxia, and we systemically profiled the expression of noncoding RNAs of DPSCs in hypoxic environments, which exhibited that a part of certain miRNAs, including miR-210-3p and miR-140-3p, were differentially upregulated under hypoxia. ${ }^{21}$ Recently, researchers have verified that miR-140-3p can increase osteocalcin (OCN) transcription and act as a key regulatory factor between wingless/integrated (Wnt) signaling and transforming growth factor-beta (TGF $\beta$ ) signaling

\footnotetext{
${ }^{1}$ Laboratory of Molecular Signaling and Stem Cells Therapy, Beijing Key Laboratory of Tooth Regeneration and Function Reconstruction, Capital Medical University School of

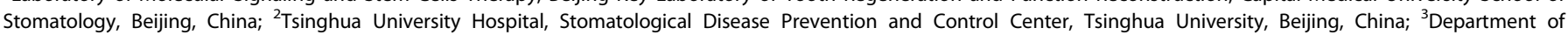

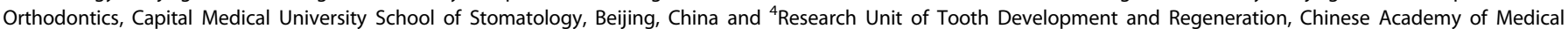
Sciences, Beijing, China

Correspondence: Haichao Jia (haichaojia@hotmail.com) or Zhipeng Fan (zpfan@ccmu.edu.cn)
}

Received: 12 June 2021 Revised: 31 October 2021 Accepted: 7 November 2021

Published online: 07 December 2021 
pathways during osteoblast differentiation. ${ }^{22}$ Another previous study pointed miR-140-3p as potential biomarkers in relation with osteoporosis. $^{23}$ Some study revealed that five miRNAs including miR-140-3p were highly expressed in standard healing fractures compared with unhealing fractures. ${ }^{24}$ Furthermore, some researchers have found that hypoxia is related with miR-140-3p, and HIF-1 $\alpha$ is identified as a target of miR-140-3p. ${ }^{25,26}$ But in fact, the role and mechanism of miR-140-3p for osteo/odontogenic differentiation of DPSCs under hypoxia have been poorly understood so far.

In this research, we observed the function of miR-140-3p for osteo/odontogenic differentiation of DPSCs under hypoxia. We found miR-140-3p upregulated the osteo/dentinogenic differentiation of DPSCs by targeting KMT5B under hypoxia condition.

\section{RESULTS}

MiR-140-3p was upregulated in $\mathrm{CoCl}_{2}$-induced hypoxia

The MSCs markers of DPSCs were verified, which showed that DPSCs were positive for cell surface markers CD90, CD105 and negative for CD34, CD45 (Supplementary Fig. 1). The hypoxia mimetic $\mathrm{CoCl}_{2}$ was used to create the hypoxia environment. Firstly, DPSCs was incubated with $0,50,100,200$, and $400 \mu \mathrm{mol} \cdot \mathrm{L}^{-1} \mathrm{CoCl}_{2}$ for $48 \mathrm{~h}$, and HIF-1 expression was obviously enhanced in the $\mathrm{CoCl}_{2}$-induced group in contrast with untreated group (Fig. 1a). Then we quantified the miR-140-3p level and the result exhibited that the miR-140-3p expression in DPSCs was dramatically upregulated in $\mathrm{CoCl}_{2}$-induced group compared with untreated group (Fig. 1b).

The miR-140-3p could enhance the osteo/odontogenic differentiation capacity of DPSCs

To observe the role of miR-140-3p in DPSCs cultured under normoxia or hypoxia, miR-140-3p inhibitor was transfected into

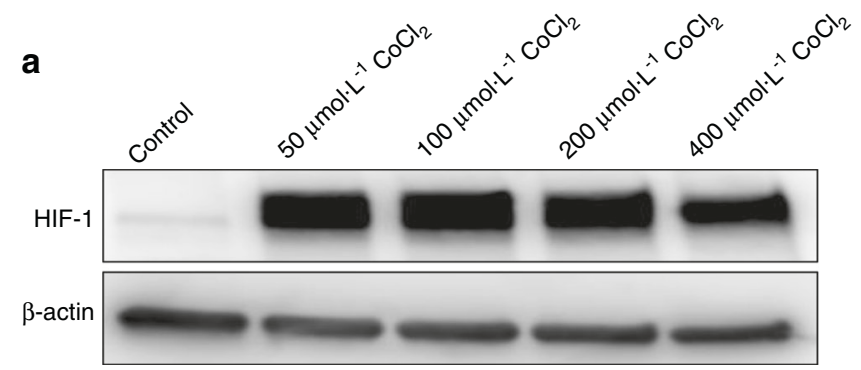

b

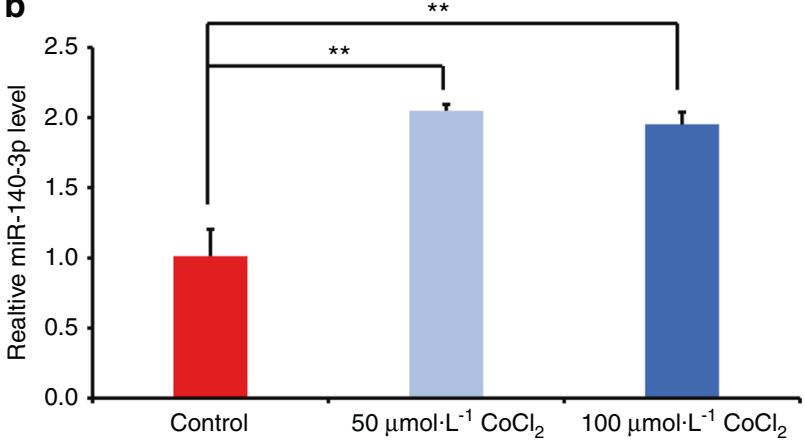

Fig. 1 MiR-140-3p was upregulated in $\mathrm{CoCl}$-induced DPSCs. a Western blot results displayed the expression of HIF-1 at $48 \mathrm{~h}$ after different dose of $\mathrm{CoCl}_{2}$ treatment. $\mathbf{b}$ The expression of miR-140$3 p$ was shown in DPSCs treated with $50,100 \mu \mathrm{mol} \cdot \mathrm{L}^{-1} \mathrm{CoCl}_{2}$, respectively. $\beta$-actin or U6 was acted as an internal control. The oneway ANOVA was applied to confirming statistical significance. Error Bars mean standard deviations $(n=3)$. ${ }^{*} P \leq 0.01$
DPSCs. After selection for 3 days by $1 \mu \mathrm{g} / \mathrm{ml}$ puromycin, the knockdown efficiency was verified by real-time RT-PCR (Fig. 2a). Then, we planned to figure out whether miR-140-3p affected the osteo/odontogenic differentiation potential of DPSCs under normoxia. After 3 days of induction, the results of ALP activity assay demonstrated that knockdown of miR-140-3p decreased ALP activity (Fig. 2b). Then after 14 days of induction, in contrast with the control group, Alizarin Red S staining and quantitative calcium measurements demonstrated suppression of mineralization in miR-140-3p-knockdown DPSCs (Fig. 2c, d). Furthermore, we detected the osteo/odontogenic-related genes, such as dentin sialophosphoprotein (DSPP), bone sialoprotein (BSP), OCN, dentin matrix acidic phosphoprotein 1 (DMP1) in 7 days of induction, and Western blot results displayed that the expression of DMP1, BSP, DSPP, and OCN were downregulated in miR-140-3p-knockdown DPSCs in contrast with the control group (Fig. 2e). Moreover, CCK8 assays showed that knockdown of miR-140-3p resulted in the number of DPSCs increased at $24 \mathrm{~h}, 48 \mathrm{~h}, 72 \mathrm{~h}$ under normoxia. And CFSE assays revealed that knockdown of miR-140-3p increased the cell proliferation at $72 \mathrm{~h}$ under normoxia (Supplementary Fig. 2).

Next, we observed the function of miR-140-3p overexpression in DPSCs. DPSCs were infected with a lentiviral miR-140-3p mimic and then selected by puromycin. The overexpression efficiency was verified using real-time RT-PCR (Fig. 3a). In three days of induction, increased ALP activity was shown in miR-140-3poverexpressing DPSCs by contrast with the control group using ALP activity assay detection (Fig. $3 \mathrm{~b}$ ). The 14 days after induction, Alizarin $S$ Red staining combining with quantitative calcium measurements also exhibited improvement of mineralization in miR-140-3p-overexpressing DPSCs compared with the control group (Fig. 3c, d). Then in 7 days of induction, results from Western blot confirmed the increasing expression of OCN, DSPP, DMP1, and BSP in miR-140-3p-overexpressing DPSCs compared with the control group (Fig. 3e).

The miR-140-3p rescued the hypoxia-induced reduction of osteo/ odontogenic differentiation potentials in DPSCs

Next, we wondered if miR-140-3p overexpressing still had the ability to enhance the osteo/odontogenic differentiation capacity of DPSCs under hypoxic condition. Thus, $50 \mu \mathrm{mol} \cdot \mathrm{L}^{-1} \mathrm{CoCl}_{2}$ was added to the osteogenic medium to mimic hypoxia environment. Surprisingly, after 3 days of induction, ALP activity assay results demonstrated that $50 \mu \mathrm{mol} \cdot \mathrm{L}^{-1} \mathrm{CoCl}_{2}$ inhibited the ALP activity of DPSCs compared with untreated group, and miR-140-3p overexpressing enhanced the decreased ALP activity in $\mathrm{CoCl}_{2}$ treated DPSCs (Fig. 3f). Besides, results from Alizarin Red $S$ staining combing with quantitative calcium measurements confirmed $50 \mu \mathrm{mol} \cdot \mathrm{L}^{-1} \mathrm{CoCl}_{2}$ suppressed the mineralization compared with untreated group, and miR-140-3p overexpressing rescued the reduced mineralization ability in $\mathrm{CoCl}_{2}$ treated DPSCs (Fig. $3 \mathrm{~g}, \mathrm{~h}$ ). Then using Western blot assay, we observed the protein levels of OCN, DSPP, DMP1, and BSP, which were decreased in $\mathrm{CoCl}_{2}$ treated DPSCs compared with untreated group, and upregulated in miR140-overexpressing DPSCs with $50 \mu \mathrm{mol} \cdot \mathrm{L}^{-1} \mathrm{CoCl}_{2}$ stimulation (Fig. 3i). And immunocytochemistry staining also verified that the protein levels of DMP1 and DSPP were downregulated in $\mathrm{CoCl}_{2}$ treated DPSCs compared with untreated group, and upregulated in miR-140-overexpressing DPSCs with $50 \mu \mathrm{mol} \cdot \mathrm{L}^{-1} \mathrm{CoCl}_{2}$ stimulation (Supplementary Fig. 3). Furthermore, CCK8 assays showed that knockdown of miR-140-3p resulted in the number of DPSCs increased at $72 \mathrm{~h}$ under hypoxia. And CFSE assays results also showed that knockdown of miR-140-3p promoted the DPSCs proliferation at $72 \mathrm{~h}$ under hypoxia (Supplementary Fig. 4).

MiR-140-3p directly inhibited KMT5B in DPSCs

To better understand the mechanism of miR-140-3p in DPSCs, then the potential targets of miR-140-3p were predicted using 


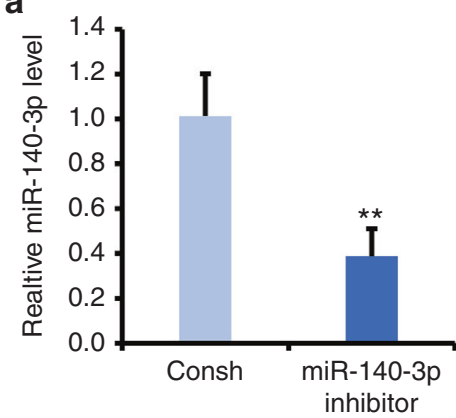

d

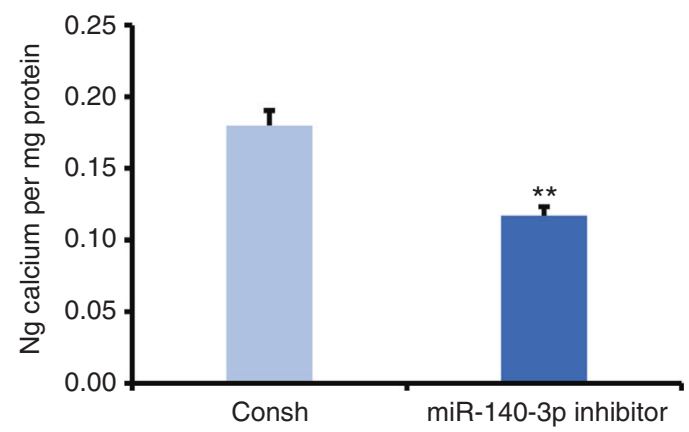

b

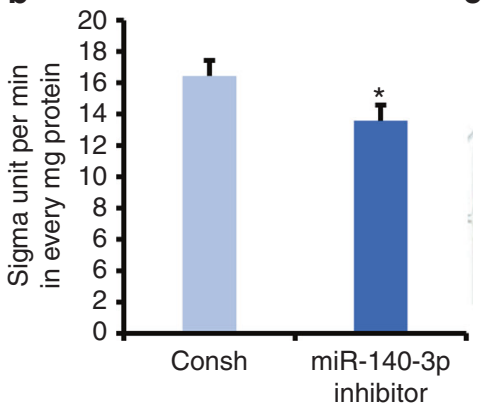

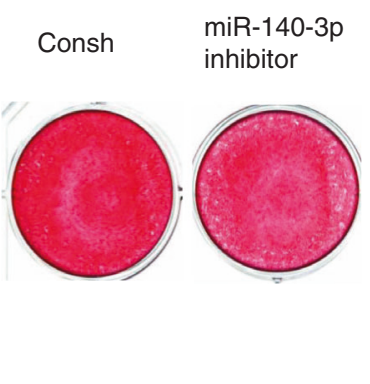

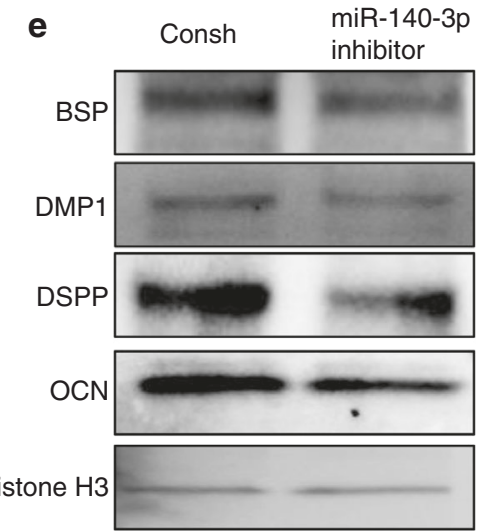

Fig. 2 Knockdown of miR-140-3p repressed the osteo/dentinogenic differentiation of DPSCs. a Real-time RT-PCR exhibited the knockdown efficiency of miR-140-3p in DPSCs. b ALP activity assay. c Alizarin Red S staining. d Quantitative calcium measurements. e The OCN, DSPP, DMP1, and BSP expressions at 7 days after the induction in DPSCs. Student's $t$-test was applied to confirming statistical significance. Histone $\mathrm{H} 3$ or U6 was acted as an internal control. Error Bars mean standard deviations $(n=3) .{ }^{*} P \leq 0.05 ;{ }^{* *} P \leq 0.01$

online target gene prediction software (miRmap, TargetScan, PITA, and PicTar online). Sirtuin-1 (SIRT1), tyrosine 3-monooxygenase/ tryptophan 5-monooxygenase activation protein gamma (YWHAG), mixed-lineage leukemia translocated to chromosome 3 protein (MLLT3), and lysine methyltransferase 5B (KMT5B) were predicted as the potential target of miR-140-3p. By real-time RTPCR, we found that SIRT1, YWHAG, and KMT5B were upregulated, and MLLT3 was downregulated in miR-140-3p-knowkdown DPSCs in contrast with the control group (Fig. 4a, b, c, d). Next, we chose one candidate target, KMT5B, to do further investigation in DPSCs. In miR-140-3p-overexpressing DPSCs, we confirmed the expression of KMT5B was decreased (Fig. 4e). Interestingly, the expression of KMT5B was also downregulated in $50 \mu \mathrm{mol} \cdot \mathrm{L}^{-1}$ $\mathrm{CoCl}_{2}$ treated DPSCs compared with untreated group (Fig. 4f). Then, by online prediction software, potential binding sites of miR$140-3 p$ in the $3^{\prime} U T R$ of KMT5B were identified (Fig. 4g). Next, we used Dual-luciferase reporter assays to verify whether KMT5B was a direct target gene of miR-140-3p. The results showed that the luciferase activity was decreased in $293 \mathrm{~T}$ cells which double transfected with miR-140-3p mimic and the wild-type KMT5B $3^{\prime}$ UTR reporter group in contrast with the control group, while the luciferase activity was no difference in $293 \mathrm{~T}$ cells which double transfected with miR-140-3p mimic and the mutant KMT5B 3'UTR reporter group compared with the control group (Fig. 4h).

KMT5B significantly suppressed the osteo/odontogenic differentiation of DPSCs

Furthermore, the function of KMT5B was investigated in DPSCs. Wild-type KMT5B construct was transfected into DPSCs. We verified the overexpression efficiency of KMT5B by Western blot (Fig. 5a). Three days after the induction, ALP activity assay results confirmed that overexpression of KMT5B inhibited ALP activity in comparison with the control group (Fig. 5b). After 14 days of induction, Alizarin Red $S$ staining and quantitative calcium measurements demonstrated that mineralization was also inhibited in KMT5B overexpressing DPSCs in contrast with the control group (Fig. 5c, d). Western blot results displayed that the expression of BSP, DSPP, DMP1, and OCN were decreased in KMT5B overexpressing DPSCs at 7 days after the induction (Fig. 5e). In addition, DPSCs were transfected with KMT5B shRNA to knock down the endogenous expression of KMT5B. The knockdown efficiency of KMT5B in DPSCs was proved to be meaningful by Western blot (Fig. 6a). Three days after osteogenic induction, we discovered that the knockdown of KMT5B obviously enhanced ALP activity compared with the control group (Fig. 6b). Then, as assessed by Alizarin Red $S$ staining along with quantitative calcium measurements, knockdown of KMT5B remarkably promoted mineralization in contrast with the control group (Fig. 6c, d). In addition, assayed by Western blot, knockdown of KMT5B significantly upregulated the expression of DMP1, OCN, DSPP, and BSP in 7 days of induction (Fig. 6e).

The depletion of KMT5B rescued the hypoxia-induced reduction of osteo/odontogenic differentiation potentials in DPSCs

Next, we wondered if KMT5B depletion had the ability to enhance the osteo/odontogenic differentiation capability of DPSCs under hypoxia. $50 \mu \mathrm{mol} \cdot \mathrm{L}^{-1} \mathrm{CoCl}_{2}$ was added to the osteogenic medium to mimic hypoxia environment. Three days after osteogenic induction, the depletion of KMT5B enhanced the decreased ALP activity in $\mathrm{CoCl}_{2}$ treated DPSCs compared with vector group (Fig. 6f). Alizarin Red S staining and quantitative calcium measurements results showed that the depletion of KMT5B rescued the reduced mineralization ability in $\mathrm{CoCl}_{2}$ treated DPSCs compared with vector group (Fig. $6 \mathrm{~g}$, h). Then Western blot results verified that OCN, DSPP, DMP1, and BSP were downregulated in $\mathrm{CoCl}_{2}$ treated DPSCs compared with untreated group, and upregulated in KMT5B depletion DPSCs with $50 \mu \mathrm{mol} \cdot \mathrm{L}^{-1} \mathrm{CoCl}_{2}$ stimulation (Fig. 6i). 
a

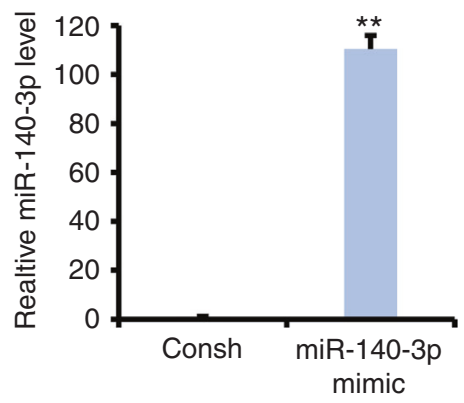

d

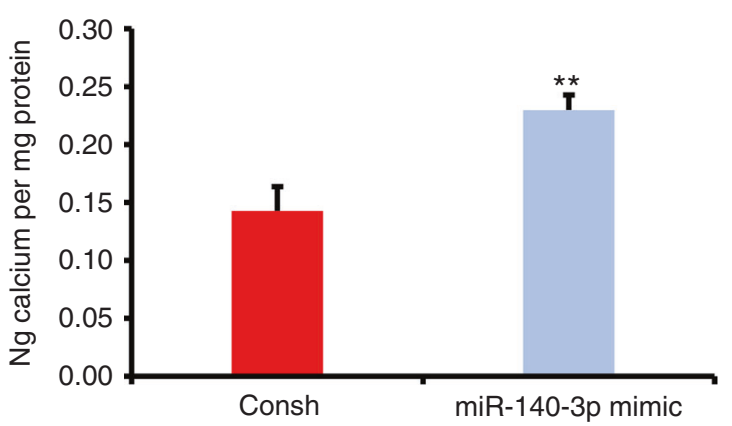

f

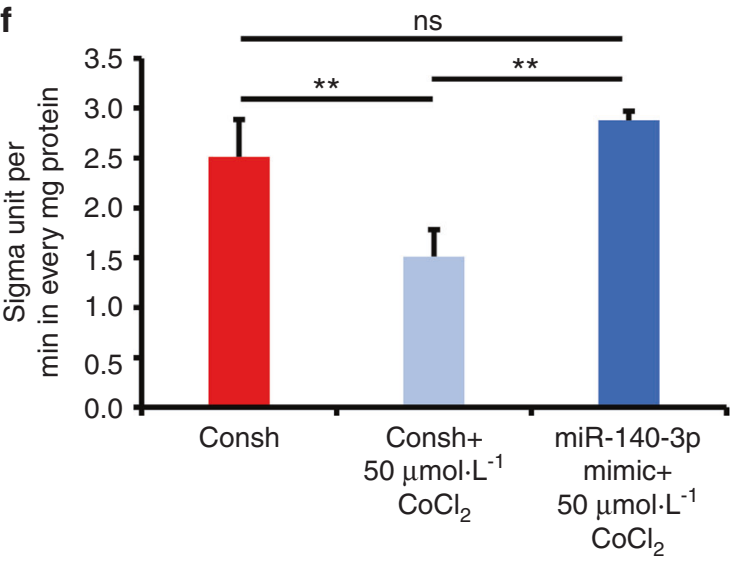

h

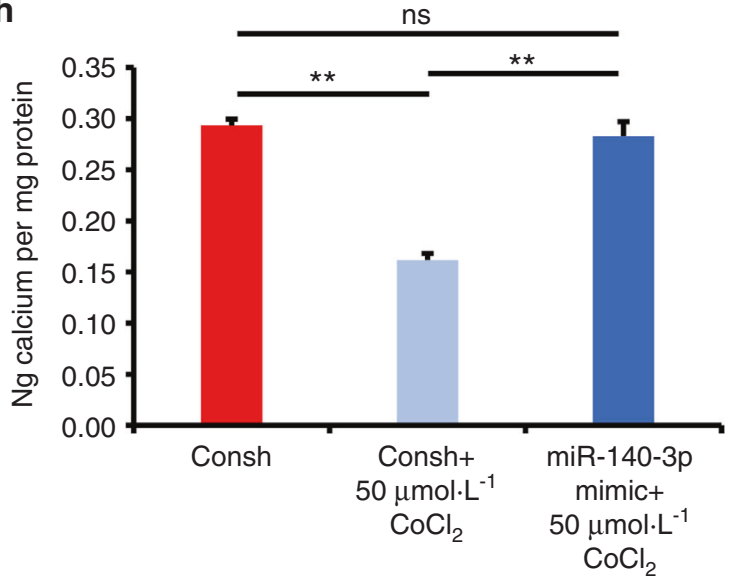

C

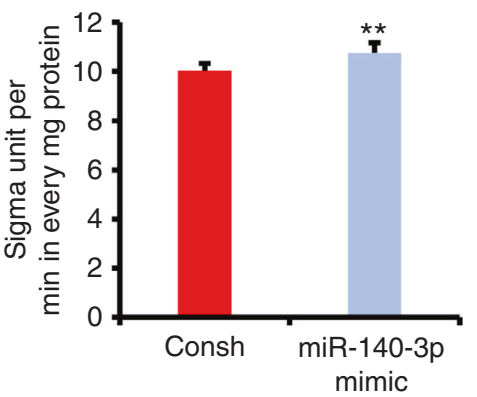

miR-140-3p

mimic

e

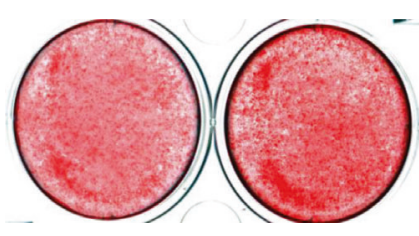

Histone $\mathrm{H} 3$

Consh

miR-140-3p mimic

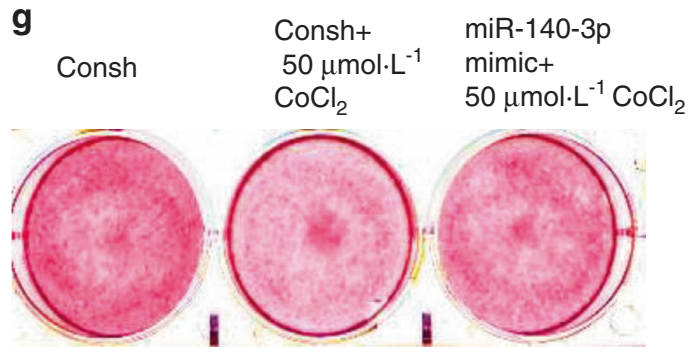

i
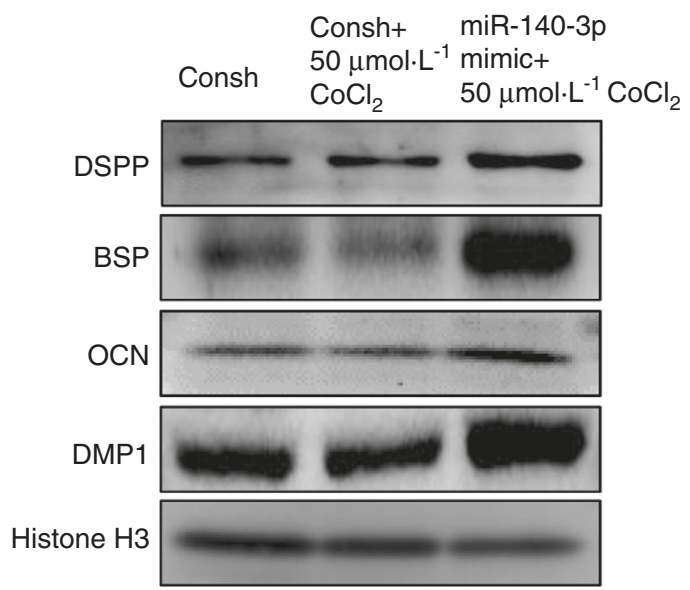

Fig. 3 Overexpression of miR-140-3p promoted the osteo/dentinogenic differentiation of DPSCs under normoxia and hypoxia. a Real-time RT-PCR exhibited the overexpression efficiency of miR-140-3p in DPSCs. b ALP activity assay. c Alizarin Red S staining. d Quantitative calcium measurements. e Western blot results of the OCN, DSPP, DMP1, and BSP expressions at 7 days after the induction in DPSCs by Western blot detection. $\mathbf{f}-\mathbf{i} 50 \mu \mathrm{mol} \cdot \mathrm{L}^{-1} \mathrm{CoCl}_{2}$ was used to treat DPSCs. $\mathbf{f}$ ALP activity assay. $\mathbf{g}$ Alizarin Red $\mathrm{S}$ staining. $\mathbf{h}$ Quantitative calcium measurements. i The OCN, DSPP, DMP1, and BSP expressions at 7 days after the induction in DPSCs by western blot detection. The one-way ANOVA or Student's $t$-test was applied to confirming statistical significance. Histone $\mathrm{H} 3$ or U6 was acted as an internal control. Error Bars mean standard deviations $(n=3)$. ${ }^{* *} P \leq 0.01$ 

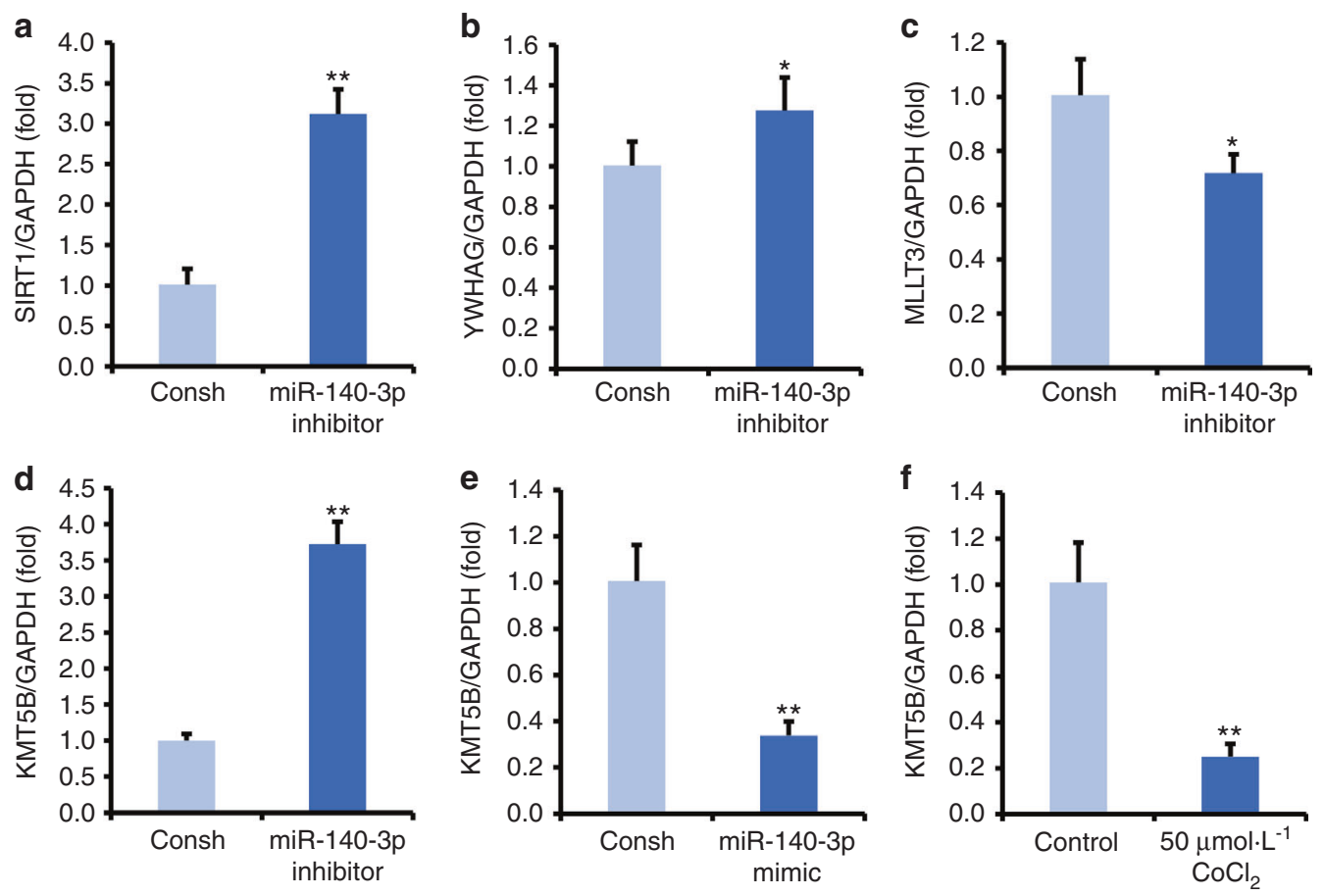

g Position 189-195 of KMT5B 3'UTR

$\operatorname{miR}-140-3 p$

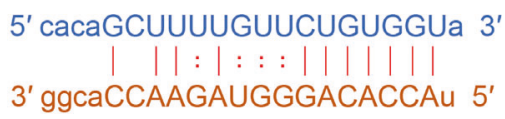

KMT5B (wt) 5' ...atgagtcacagctttgttCTGTGGTA... 3'

KMT5B (mut) $5^{\prime}$.... atgagtcacagctttgttTCGAAACC ... 3'

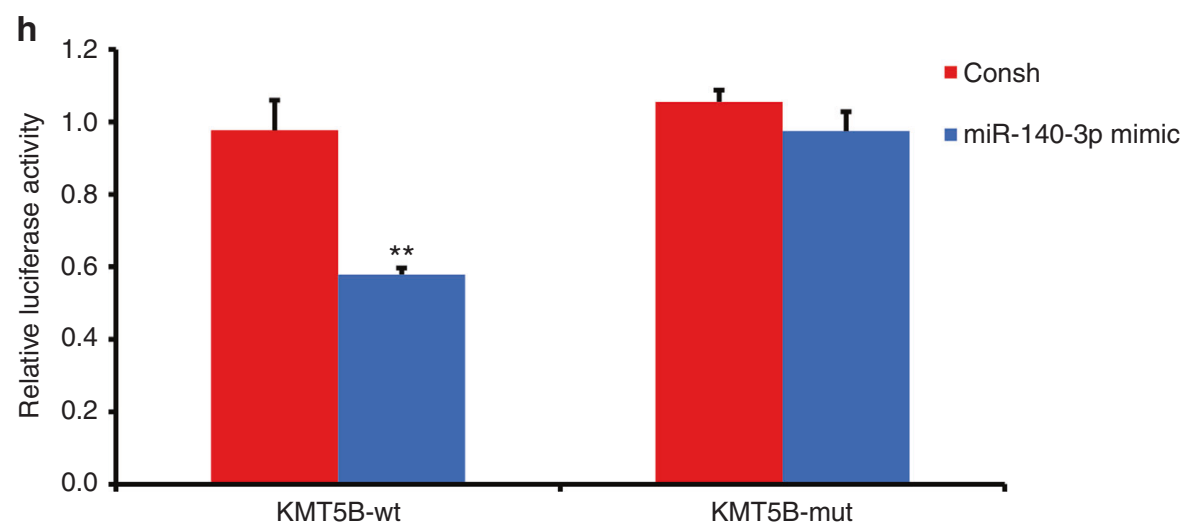

Fig. 4 MiR-140-3p negatively regulated KMT5B in DPSCs. a-d DPSCs were transfected with a negative control (Consh) or miR-140-3p inhibitor, and SIRT1 (a), YWHAG (b), MLLT3 (c), KMT5B (d) expressions were measured by real-time RT-PCR. e DPSCs were transfected with a negative control (Consh) or miR-140-3p mimic, and KMT5B expression was verified by real-time RT-PCR. $\mathbf{f}$ The KMT5B expression was shown in DPSCs treated with $50 \mu \mathrm{mol} \cdot \mathrm{L}^{-1} \mathrm{CoCl}_{2}$. g Predicted binding site of miR-140-3p on 3'UTR of KMT5B. $\mathbf{h}$ Dual-luciferase reporter assays. Student's $t$-test was applied to confirming statistical significance. GAPDH was acted as an internal control. Error Bars mean standard deviations $(n=3)$. ${ }^{*} P \leq 0.05 ;{ }^{* *} P \leq 0.01$

\section{DISCUSSION}

The hypoxia microenvironment has been reported to affect the function and the curative effect of MSCs, and we aim to investigate the molecular mechanism of MSCs under hypoxia to find potential target for improving MSCs function. ${ }^{27-29}$ Emerging evidence showed that miRNAs were widely involved in a variety of biological functions such as proliferation, apoptosis and differentiation of MSCs under the condition of hypoxia. ${ }^{30-33}$ Our previous result displayed that miR-140-3p was upregulated in DPSCs under $3 \%$ hypoxia. $^{21}$ And in this research, we further investigated the role of miR-140-3p in DPSCs under hypoxia. Cobalt chloride $\left(\mathrm{CoCl}_{2}\right)$ has been seen as a useful hypoxia mimetic, inhibiting the formation of oxygenated hemoglobin. ${ }^{34,35}$ During cell culture, $\mathrm{CoCl}_{2}$ repressed the catalysis of prolyl hydroxylases, creating an intracellular hypoxia-like state. ${ }^{36}$ Hence, $\mathrm{CoCl}_{2}$ was applied in this research to mimic hypoxia. $\mathrm{CoCl}_{2}$ has been seen as a hypoxia-mimicking agent to create the hypoxia condition via inducing the accumulation of hypoxia-inducible factor-1 (HIF-1), an oxygen-sensitive transcriptional activator. ${ }^{37-39}$ The HIF-1 is a critical oxygen-sensitive transcriptional activator, which is 


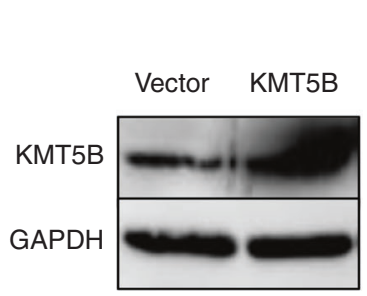

d

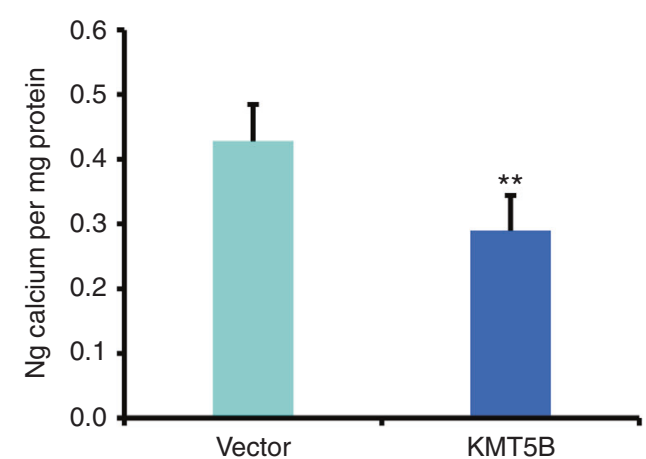

b

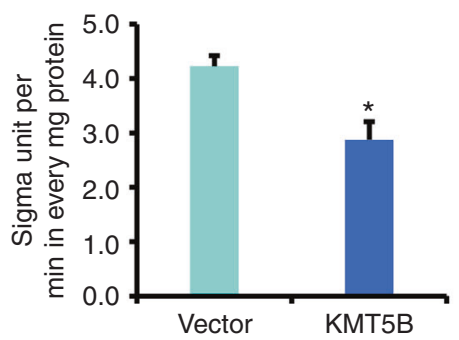

C
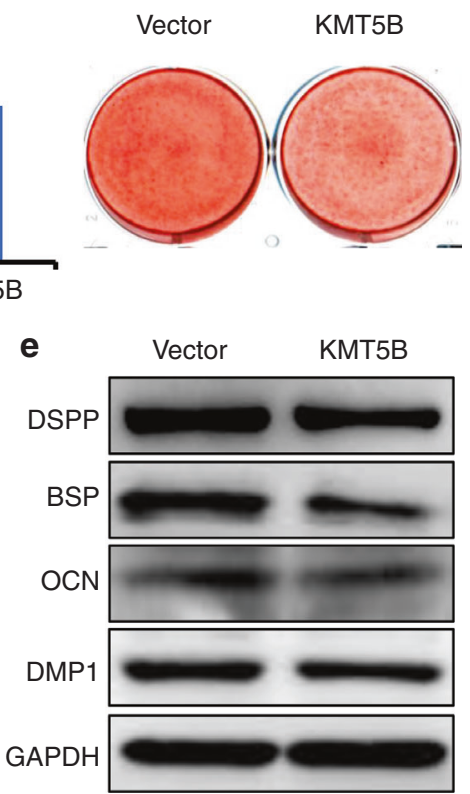

Fig. 5 Overexpression of KMT5B repressed the osteo/dentinogenic differentiation of DPSCs. a Western blot exhibited the overexpression of KMT5B in DPSCs. b ALP activity assay. c Alizarin Red S staining. d Quantitative calcium measurements. e The OCN, DSPP, DMP1, and BSP expressions at 7 days after the induction in DPSCs by western blot detection. Student's $t$-test was applied to confirming statistical significance. GAPDH was acted as an internal control. Error Bars mean standard deviations $(n=3)$. ${ }^{*} P \leq 0.05 ;{ }^{* *} P \leq 0.01$

hydroxylated and becomes proteasomal degradation at higher oxygen tensions, but under hypoxia, HIF-1 becomes more stable, and serves as transcription factors. ${ }^{40-42}$ In the present research, we detected the influence of $\mathrm{CoCl}_{2}$ on HIF-1 expression at different dose, and found that $50 \mu \mathrm{mol} \cdot \mathrm{L}^{-1}, 100 \mu \mathrm{mol} \cdot \mathrm{L}^{-1}, 200 \mu \mathrm{mol} \cdot \mathrm{L}^{-1}$, and $400 \mu \mathrm{mol} \cdot \mathrm{L}^{-1} \mathrm{CoCl}_{2}$ had a similar effect on HIF-1 expression. Then, we used $50 \mu \mathrm{mol} \cdot \mathrm{L}^{-1}$ and $100 \mu \mathrm{mol} \cdot \mathrm{L}^{-1} \mathrm{CoCl}_{2}$ to treat DPSCs, and verified that miR-140-3p expression was upregulated as same level in $50 \mu \mathrm{mol} \cdot \mathrm{L}^{-1}$ and $100 \mu \mathrm{mol} \cdot \mathrm{L}^{-1} \mathrm{CoCl}_{2}$ treated DPSCs, which meant $\mathrm{CoCl}_{2}$-induced hypoxia increased miR-140-3p expression, which was consistent with our previous study. Considering the toxicity of $\mathrm{CoCl}_{2}$, we chose $50 \mu \mathrm{mol} \cdot \mathrm{L}^{-1} \mathrm{CoCl}_{2}$ to mimic hypoxia in the following experiment, and we found that $50 \mu \mathrm{mol} \cdot \mathrm{L}^{-1} \mathrm{CoCl}_{2}$ repressed the differentiation capacity of DPSCs by detecting ALP activity, mineralization in vitro, and the indicators of osteo/odontogenic differentiation including DMP1, OCN, DSPP, and BSP, which was supported by our previous research that hypoxia $\left(3 \% \mathrm{O}_{2}\right)$ repressed the osteo/odontogenic differentiation capacity of DPSCs. ${ }^{21}$

Then, we performed gain- and loss- of- function assays to test the function of miR-140-3p. Results from ALP activity, mineralization in vitro, and the expression of DMP1, OCN, BSP, and DSPP comprehensively clarified the knockdown of miR-140-3p restrained the osteo/dentinogenic differentiation of DPSCs. Accordingly, the overexpression of miR-140-3p facilitated osteo/ dentinogenic differentiation under normoxia. Furthermore, we found that the overexpression of miR-140-3p reversed the suppression in osteo/odontogenic differentiation of DPSCs caused by $\mathrm{CoCl}_{2}$-induced hypoxia. There were lots of factors that could affect differentiation of DPSCs under hypoxia, some genes play positive effect, some genes play negative effect. But totally effect of genes caused osteo/odontogenic differentiation of DPSCs decreased under hypoxic conditions. In present study, it was found that miR-140-3p enhanced the osteo/odontogenic differentiation of DPSCs, and hypoxia environment upregulated the expression of miR-140-3p in DPSCs. It indicated that hypoxia environment upregulated the expression of miR-140-3p, but the upregulated endogenous miR-140-3p could not completely antagonize the negative effect of other genes, so the phenotype in DPSCs was still decreased osteo/odontogenic differentiation. And these suggested that we need more miR-140-3p to antagonize the negative effect in hypoxia environment. Indeed, when we over-expressed exogenous miR-140-3p in DPSCs, they could completely antagonize the negative effect of other genes, and the phenotype in DPSCs became enhanced osteo/odontogenic differentiation. These results verified overexpression of miR140-3p could enhance the osteo/odontogenic differentiation of DPSCs under hypoxia condition, which indicated that miR-140-3p might have a meaningful effect on hypoxia, suggesting miR-140$3 p$ as a novel target for dental tissue regeneration.

Furthermore, we observed the mechanism of miR-140-3p in DPSCs. MiRNAs worked post-transcriptionally by mainly basepairing to the 3 '-untranslated regions of targets genes. ${ }^{43}$ Therefore, a promising target of miR-140-3p is needed for further investigating in DPSCs. Researchers have verified some targets of miR-140-3p. Some studies reported that miR-140-3p was involved in osteoblast differentiation as a critical regulatory factor, and demonstrated TGF 33 acted as a direct target of miR-140-3p. ${ }^{22}$ Moreover, miR-140-3p had inhibitory effect on preosteoblast viability and differentiation via targeting MCF2L in osteoporosis. ${ }^{44}$ In the stem cell differentiation, some reported that miR-140-3p negatively regulated osteogenic differentiation of BMSCs, which could be reversed by Spred2. ${ }^{45}$ Besides, miR-140 targeted RALA and regulated chondrogenic differentiation of MSCs by translational enhancement of SOX9 and ACAN. ${ }^{46}$ In present study, we used the TargetScan, miRmap, PITA, and PicTar software to predict the potential targets of miR-140-3p. SIRT1, YWHAG, MLLT3, and KMT5B were predicted as the potential target of miR-140-3p. Further we found that knockdown of miR-140-3p upregulated SIRT1, YWHAG, and KMT5B, while downregulated MLLT3 at mRNA level in DPSCs. It was reported that SIRT1, a NAD-dependent histone deacetylase, played a positive role in osteogenic 
a

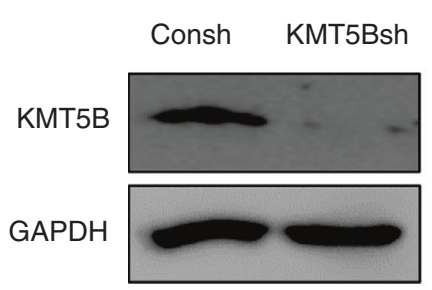

b

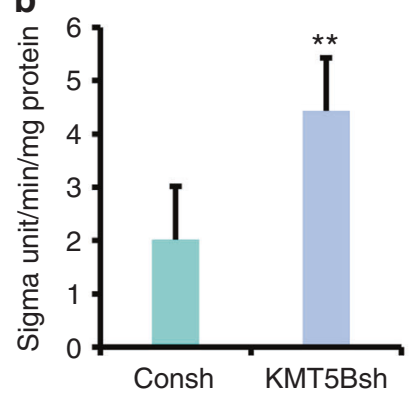

C

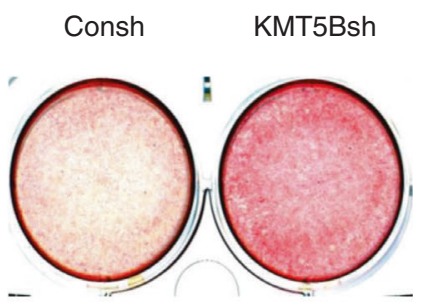

d

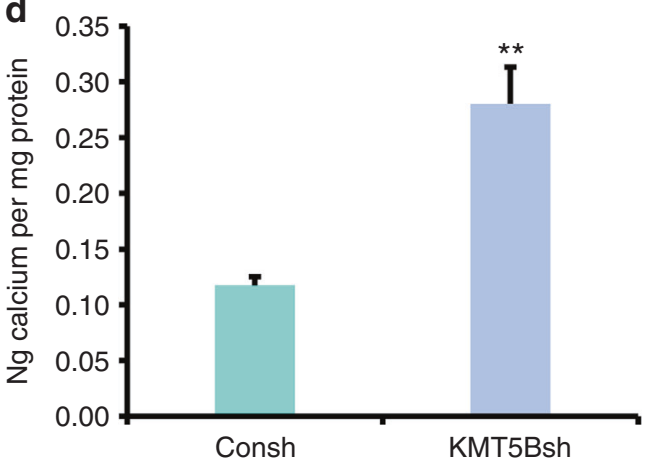

f

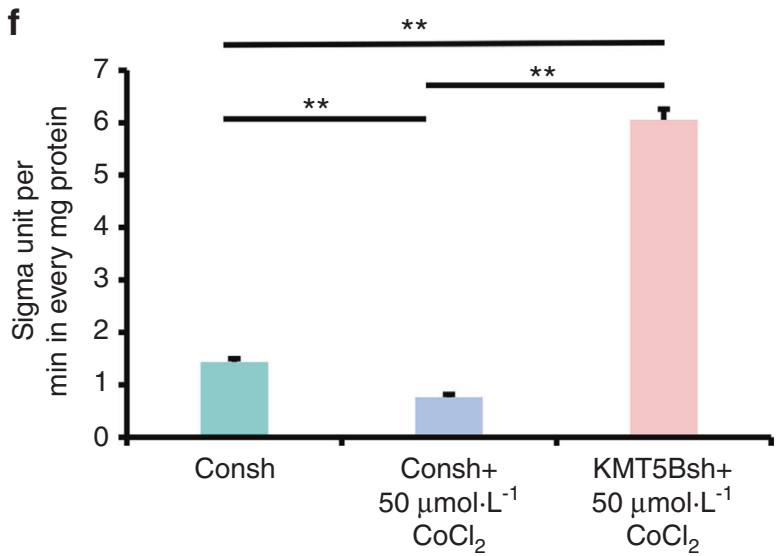

h

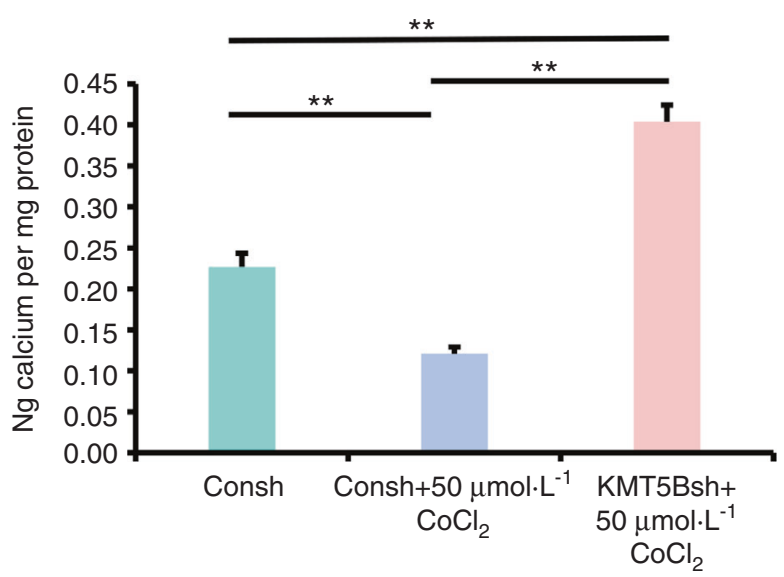

e

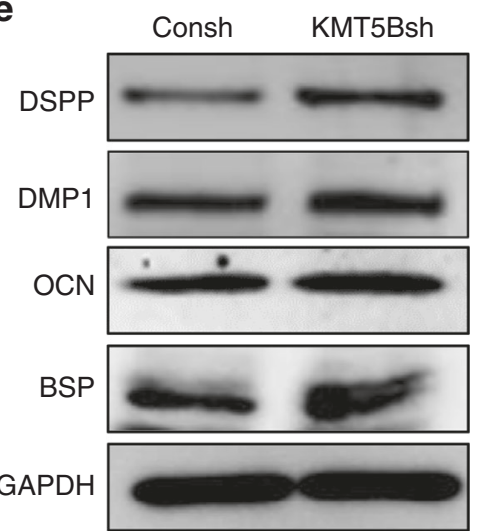

g Consh+ KMT5Bsh+ Consh $\quad 50 \mu \mathrm{mol} \cdot \mathrm{L}^{-1} \quad 50 \mu \mathrm{mol} \cdot \mathrm{L}^{-1}$ $\mathrm{CoCl}_{2} \quad \mathrm{CoCl}_{2}$

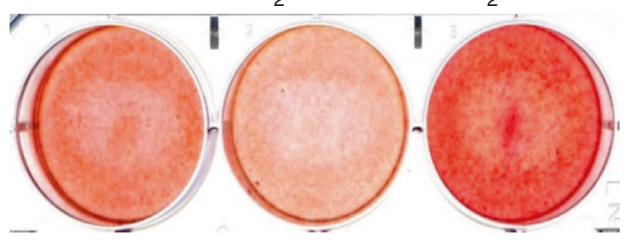

i
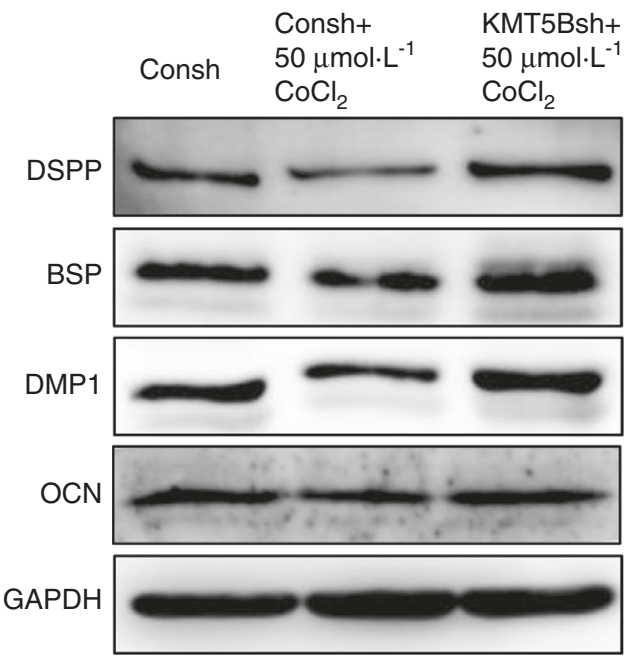

Fig. 6 The depletion of KMT5B enhanced the osteo/dentinogenic differentiation of DPSCs under normoxia and hypoxia. a The knockdown efficiency of KMT5B by western blot detection. b ALP activity assay. c Alizarin Red S staining. d Quantitative calcium measurements. e The OCN, DSPP, DMP1, and BSP expressions at 7 days after the induction in DPSCs by western blot detection. $\mathbf{f}-\mathbf{i} 50 \mu \mathrm{mol} \cdot \mathrm{L}^{-1} \mathrm{CoCl} \mathrm{C}_{2}$ was used to treat DPSCs. f ALP activity assay. g Alizarin Red S staining. $\mathbf{h}$ Quantitative calcium measurements. $\mathbf{i}$ The OCN, DSPP, DMP1, and BSP expressions at 7 days after the induction in DPSCs by western blot detection. The one-way ANOVA or Student's $t$-test was applied to confirming statistical significance. GAPDH was acted as an internal control. Error Bars mean standard deviations $(n=3)$. ${ }^{* *} P \leq 0.01$ 
differentiation of DPSCs treated by TNF-a. ${ }^{47}$ In the process of serial subculture during osteogenic differentiation of MSCs, some researchers found a number of differentially regulated proteins. It was showed that 14-3-3 protein gamma (YWHAG) was upregulated during the later passage, which provided some clues for regulating the osteogenic differentiation process. ${ }^{48}$ Histone modifications including methylation of key lysine residues play a crucial role in lots of cellular events. ${ }^{49}$ KMT5B encodes a protein containing a SET domain, belonging to one group of histone methyltransferases (KMTs). Although the function of KMT5B in stem cells was little known, it has been reported that certain genes of the KMT family could regulate the osteo/odontogenic differentiation of dental tissue derived MSCs. ${ }^{50,51}$ Enhancer of zeste homolog 2 (EZH2), also known as KMT6A, was one of widely studied SET domain-containing KMTs concerning MSC differentiation. ${ }^{52}$ EZH2 was a methyltransferase for $\mathrm{H} 3$ lysine 27 trimethylation, which could suppress osteogenic differentiation of DPSCs while promoting proliferation, indicating its role in dental pulp regeneration. ${ }^{53}$ Thus, we selected KMT5B for further study. And we found the expression of KMT5B was reversed by miR-140-3p and reversely correlated with miR-140-3p in $\mathrm{CoCl}_{2}$-induced hypoxia, which indicated that KMT5B might be the key target of miR-140$3 p$ under hypoxia. Furthermore, using dual-luciferase reporter assay, we verified miR-140-3p binding into the binding element of the KMT5B 3'UTR region. These results demonstrated miR-140-3p directly targeted KMT5B in DPSCs.

Then we wondered whether miR-140-3p affected the osteo/ odontogenic differentiation via KMT5B, and investigated the function of KMT5B in DPSCs. The gain- and loss- of- function assay results exhibited that KMT5B repressed the osteo/odontogenic differentiation of DPSCs showing by ALP activity, mineralization in vitro, and the protein levels of DMP1, BSP, OCN, and DSPP. Interestingly, the knockdown of KMT5B remarkably enhanced the hypoxia-impaired differentiation capacity of DPSCs. Taken together, these evidences indicated that miR-140-3p could increase the impaired osteo/odontogenic differentiation capacity of DPSCs under hypoxic conditions via directly targeting KMT5B.

\section{CONCLUSION}

To sum up, in present study, the miR-140-3p could restore impaired osteo/odontogenic differentiation potentials of DPSCs under hypoxic conditions. Moreover, this study explored that miR$140-3 p$ directly targeted KMT5B, and the depletion of KMT5B could remarkably rescue the impaired osteo/odontogenic differentiation of DPSCs under hypoxic conditions. These suggested that miR-140-3p enhanced the hypoxia-impaired osteo/odontogenic differentiation potentials of DPSCs via negatively regulating KMT5B, revealed a new mechanism for regulating osteo/dentinogenic differentiation of DPSCs in a hypoxic microenvironment, and provided the potential targets miR-140-3p and KMT5B for DPSCmediated tissue regeneration.

\section{MATERIALS AND METHODS}

DPSC culture and drug administration

Dental pulps were physically obtained from the crown and root and then subjected to a collagenase and dispase digest. Simply, we acquired, cultured, and then identified DPSCs as previously stated. ${ }^{54}$ DPSCs from young group at passages 3-5 were used in this study.

To mimic the hypoxia condition, $\mathrm{CoCl}_{2}$ was diluted to $50 \mu \mathrm{mol} \cdot \mathrm{L}^{-1}, 100 \mu \mathrm{mol} \cdot \mathrm{L}^{-1}, 200 \mu \mathrm{mol} \cdot \mathrm{L}^{-1}, 400 \mu \mathrm{mol} \cdot \mathrm{L}^{-1}$ for actual use. In order to induce osteo/odontogenic differentiation, DPSCs were plated at a density of $2 \times 10^{5}$ cells per plate in six-well plates. Then DPSCs were changed into the differentiation medium containing $\beta$-glycerophosphate, dexamethasone and inorganic phosphate after cells reached $80 \%$ confluence.
Viral infection

MiR-140-3p inhibitor, miR-140-3p mimic, KMT5B shRNA and control shRNA (Consh) lentivirus were all designed and synthesized by GenePharma (Suzhou, China). The miR-140-3p inhibitor sequence was $5^{\prime}$-CCGTGGTTCTACCCTGTGGTA-3', the miR-140-3p mimic sequence was 5'-TACCACAGGGTAGAACCACGG-3', KMT5B shRNA (KMT5Bsh) sequence was 5'-GGAGAAATGGAGGCAAGTTG T- $3^{\prime}$ and the control shRNA sequence was $5^{\prime}-T$ TCTCCGAACGTG TCACGT-3'. Besides, KMT5B and Vector were also purchased from GenePharma. Abiding by the manufacturer's protocol, DPSCs transfected with lentiviruses lasted $12 \mathrm{~h}$ along with $6 \mathrm{mg} / \mathrm{ml}$ polybrene. Then infected cells were selected by $1 \mu \mathrm{g} \cdot \mathrm{mL}^{-1}$ puromycin.

Alkaline phosphatase (ALP) activity and Alizarin Red S staining About the ALP activity assay, DPSCs were seeded in six-well plates and changed to osteogenic differentiation medium for 3 days. Next, in order to confirm mineralization, DPSCs were induced for 14 days, and we used $70 \%$ ethanol to fix these cells. $2 \%$ Alizarin red was then applied to staining the formation of mineralized nodules, as previously stated. ${ }^{55}$

RNA extraction and real-time RT-PCR

Trizol reagent (Invitrogen) was used to extract total RNA from DPSCs. The levels of miR-140-3p were observed as previously stated. ${ }^{56}$ Besides, following the manufacturer's protocol (Invitrogen), $2 \mu \mathrm{g}$ RNA sample was reverse transcribed into cDNA. The KMT5B primers are as follows: forward, 5'-GAGAAATGGAGGC AAGTTGTCT-3'; and reverse, 5'-ACATAGCGACTCTGTCCTTCA-3'. The SIRT1 primers are as follows: forward, 5'-TAGCCTTGTCAGAT AAGGAAGGA-3'; and reverse, 5'-ACAGCTTCACAGTCAACTITGT-3'. The YWHAG primers are as follows: forward, 5'-AGCCACTGTCG AATGAGGAAC-3'; and reverse, 5'-CTGCTCAATGCTACTGATGACC-3'. The MLLT3 primers are as follows: forward, 5'-TाTGTGGAGA AAGTCGTCTTCC-3'; and reverse, 5'-GAGGTGATTCACTGGTGGAT G-3'. The GAPDH primers are as follows: forward, 5'-CGGACCAAT ACGACCAAATCCG-3'; reverse, 5'-AGCCACATCGCTCAGACACC-3'.

\section{Western blot}

Total proteins were extracted from DPSCs using Radio immunoprecipitation assay (RIPA) lysis buffer and protease inhibitors. $25 \mu \mathrm{g}$ protein from each sample was prepared for Western blot analysis, which was performed as previously stated. ${ }^{57}$ The primary antibodies used in this study are as follows: DMP1 (Cat No. bs12359R, Bioss), HIF-1 (Cat No. 36169, Cell Signaling Technology), Histone H3 (Cat No.17168-1-AP, proteintech), OCN (Cat No. bs4917R, Bioss), KMT5B (Cat No. ab118659, abcam), DSPP (Cat No. bs10316R, Bioss), GAPDH (Cat No. 60004-1-lg, proteintech), $\beta$-actin (Cat No. C1313; Applygen, China), BSP (Cat No. bs2668R, Bioss).

\section{Dual-luciferase reporter assays}

Before transfection, $293 \mathrm{~T}$ cells $\left(1.0 \times 10^{5}\right.$ cells per well) were seeded in 12-well plates and incubated overnight. MiR-140-3p mimic or mimic control along with the wild-type (wt) or mutant (mut) KMT5B 3'UTR-Luc reporter construct was then cotransfected into $293 \mathrm{~T}$ cells, as previously stated. ${ }^{56} 48 \mathrm{~h}$ later, luciferase enzymatic activities were then detected using the DualLuciferase Reporter Assay Kit (Vazyme).

\section{Cell Counting Kit-8 (CCK8) Assay}

CCK8 assay (MCE, Cat. HY-K0301) was used to observe cell proliferation. DPSCs $\left(5 \times 10^{3}\right.$ cells per well) were seeded into $96-$ well plates. At $24 \mathrm{~h}, 48 \mathrm{~h}$ or $72 \mathrm{~h}$, the culture medium was removed and changed into $100 \mu \mathrm{L}$ MEM mixed with $10 \mu \mathrm{L}$ CCK8. And the plate was incubated for $1 \mathrm{~h}$ at $37^{\circ} \mathrm{C}$. A multiwell spectrophotometer was used to detected absorbance (OD) at $450 \mathrm{~nm}$. 
Carboxyfluorescein succinimidyl ester (CFSE) assay In short, DPSCs were labelled as previously depicted..$^{56}$ A flow cytometry was then used to detect the labeling cells 3 days later. The data was analyzed using MODFIT LT.

\section{Immunocytochemical staining}

The DPSCs were seeded on coverslips in 24-well plates. $24 \mathrm{~h}$ later, the DPSCs were changed into the differentiation medium and meanwhile $50 \mu \mathrm{mol} \cdot \mathrm{L}^{-1} \mathrm{CoCl}_{2}$ was added into $\mathrm{CoCl}_{2}$ treated group. After 7 days osteo/odontogenic induction, the DPSCs were fixed for about $30 \mathrm{~min}$. Simply, after incubated in $3 \% \mathrm{H}_{2} \mathrm{O}_{2}$ for $10 \mathrm{~min}$ followed by three PBS washes, goat serum was applied. Importantly, the primary antibodies were as follows: DSPP polyclonal antibody (1:100), DMP1 polyclonal antibody (1:100). Finally, the cells were counterstained with haematoxylin and observed under a microscope.

Statistics analysis

SPSS 10 software was used to analyze data. One-way ANOVA or student's $t$-test was used to assess the statistical differences, with a $P$-value $\leq 0.05$ deemed to be significant.

\section{ACKNOWLEDGEMENTS}

This work was supported by grants from the National Natural Science Foundation of China (81625005 to Z.P.F.), CAMS Innovation Fund for Medical Sciences (2019-I2M-5031 to Z.P.F.), the Program for "Hundred-Thousand-Ten Thousand" Talents in Beijing (2018A16 to Z.P.F.), Beijing Key Laboratory of Tooth Regeneration and Function Reconstruction (KFKT2019012 to L.L).

\section{ADDITIONAL INFORMATION}

Supplementary information The online version contains supplementary material available at https://doi.org/10.1038/s41368-021-00148-y.

Competing interests: The authors declare no competing interests.

\section{REFERENCES}

1. AlRahabi, M. K. Evaluation of complications of root canal treatment performed by undergraduate dental students. Libyan J. Med. 12, 1345582 (2017).

2. Saoud, T. M., Mistry, S., Kahler, B., Sigurdsson, A. \& Lin, L. M. Regenerative endodontic procedures for traumatized teeth after horizontal root fracture, avulsion, and perforating root resorption. J. Endod. 42, 1476-1482 (2016).

3. Botelho, J., Cavacas, M. A., Machado, V. \& Mendes, J. J. Dental stem cells: recent progresses in tissue engineering and regenerative medicine. Ann. Med. 49, 644-651 (2017).

4. Ouchi, T. \& Nakagawa, T. Mesenchymal stem cell-based tissue regeneration therapies for periodontitis. Regen. Ther. 14, 72-78 (2020).

5. Andrukhov, O., Behm, C., Blufstein, A. \& Rausch-Fan, X. Immunomodulatory properties of dental tissue-derived mesenchymal stem cells: Implication in disease and tissue regeneration. World J. Stem Cells 11, 604-617 (2019).

6. Zheng, C., Chen, J., Liu, S. \& Jin, Y. Stem cell-based bone and dental regeneration: a view of microenvironmental modulation. Int. J. Oral. Sci. 11, 23 (2019).

7. Wobma, H. M. et al. The influence of hypoxia and IFN- $\gamma$ on the proteome and metabolome of therapeutic mesenchymal stem cells. Biomaterials 167, 226-234 (2018).

8. Hu, H. M. et al. Artemisinin protects DPSC from hypoxia and TNF-a mediated osteogenesis impairments through CA9 and Wnt signaling pathway. Life Sci. 277, 119471 (2021).

9. Liu, Z. et al. Transforming growth factor- $\beta 1$ and hypoxia inducible factor- $1 a$ synergistically inhibit the osteogenesis of periodontal ligament stem cells. Int. Immunopharmacol. 75, 105834 (2019).

10. lida, K. et al. Hypoxia enhances colony formation and proliferation but inhibits differentiation of human dental pulp cells. Arch. Oral. Biol. 55, 648-654 (2010)

11. Zhang, Q. B. et al. Effects of hypoxia on proliferation and osteogenic differentiation of periodontal ligament stem cells: an in vitro and in vivo study. Genet Mol. Res. 13, 10204-10214 (2014).

12. Ito, K., Matsuoka, K., Matsuzaka, K., Morinaga, K. \& Inoue, T. Hypoxic condition promotes differentiation and mineralization of dental pulp cells in vivo. Int. Endod. J. 48, 115-123 (2015).
13. Hass, R., Kasper, C., Böhm, S. \& Jacobs, R. Different populations and sources of human mesenchymal stem cells (MSC): a comparison of adult and neonatal tissue-derived MSC. Cell Commun. Signal 9, 12 (2011).

14. Mens, M. M. J. \& Ghanbari, M. Cell cycle regulation of stem cells by MicroRNAs. Stem Cell Rev. Rep. 14, 309-322 (2018).

15. Kaneda-lkeda, E. et al. Periodontal ligament cells regulate osteogenesis via miR299-5p in mesenchymal stem cells. Differentiation 112, 47-57 (2020).

16. Chang, W. et al. Survival of hypoxic human mesenchymal stem cells is enhanced by a positive feedback loop involving miR-210 and hypoxia-inducible factor 1 . J. Vet. Sci. 14, 69-76 (2013).

17. Zhang, Y. et al. microRNA-206 is involved in survival of hypoxia preconditioned mesenchymal stem cells through targeting Pim-1 kinase. Stem Cell Res. Ther. 7, 61 (2016).

18. Ching, H. S., Luddin, N., Rahman, I. A. \& Ponnuraj, K. T. Expression of odontogenic and osteogenic markers in DPSCs and SHED: a review. Curr. Stem Cell Res. Ther 12, 71-79 (2017).

19. Gronthos, S. et al. Stem cell properties of human dental pulp stem cells. J. Dent. Res. 81, 531-535 (2002).

20. Sui, B. et al. Dental pulp stem cells: from discovery to clinical application. J. Endod. 46, S46-s55 (2020).

21. Shi, R. et al. Analysis of the characteristics and expression profiles of coding and noncoding RNAs of human dental pulp stem cells in hypoxic conditions. Stem Cell Res. Ther. 10, 89 (2019).

22. Fushimi, S., Nohno, T., Nagatsuka, H. \& Katsuyama, H. Involvement of miR-140-3p in Wnt3a and TGF 33 signaling pathways during osteoblast differentiation in MC3T3-E1 cells. Genes Cells 23, 517-527 (2018).

23. Ramírez-Salazar, E. G. et al. Serum miRNAs miR-140-3p and miR-23b-3p as potential biomarkers for osteoporosis and osteoporotic fracture in postmenopausal Mexican-Mestizo women. Gene 679, 19-27 (2018).

24. Waki, T. et al. Profiling microRNA expression during fracture healing. BMC Musculoskelet. Disord. 17, 83 (2016).

25. Liang, S. et al. LncRNA SNHG1 alleviates hypoxia-reoxygenation-induced vascular endothelial cell injury as a competing endogenous RNA through the HIF-1a/VEGF signal pathway. Mol. Cell Biochem. 465, 1-11 (2020).

26. Chen, F. et al. Hypoxia induced changes in miRNAs and their target mRNAs in extracellular vesicles of esophageal squamous cancer cells. Thorac. Cancer 11, 570-580 (2020).

27. Chen, J. et al. Mesenchymal stem cell-derived exosomes protect beta cells against hypoxia-induced apoptosis via miR-21 by alleviating ER stress and inhibiting p38 MAPK phosphorylation. Stem Cell Res. Ther. 11, 97 (2020).

28. $\mathrm{Hu}, \mathrm{C}$. \& Li, L. Preconditioning influences mesenchymal stem cell properties in vitro and in vivo. J. Cell Mol. Med. 22, 1428-1442 (2018).

29. Wagegg, M. et al. Hypoxia promotes osteogenesis but suppresses adipogenesis of human mesenchymal stromal cells in a hypoxia-inducible factor-1 dependent manner. PLoS ONE 7, e46483 (2012).

30. Liu, W. et al. Hypoxic mesenchymal stem cell-derived exosomes promote bone fracture healing by the transfer of miR-126. Acta Biomater. 103, 196-212 (2020).

31. Qu, H. M., Qu, L. P., Pan, X. Z. \& Mu, L. S. Upregulated miR-222 targets BCL2L11 and promotes apoptosis of mesenchymal stem cells in preeclampsia patients in response to severe hypoxia. Int. J. Clin. Exp. Pathol. 11, 110-119 (2018).

32. Fan, J. et al. MiR-1292 targets FZD4 to regulate senescence and osteogenic differentiation of stem cells in TE/SJ/mesenchymal tissue system via the Wnt/ ß-catenin pathway. Aging Dis. 9, 1103-1121 (2018).

33. Wang, B., Gu, T. X., Yu, F. M., Zhang, G. W. \& Zhao, Y. Overexpression of miR-210 promotes the potential of cardiac stem cells against hypoxia. Scand. Cardiovasc. J. 52, 367-371 (2018)

34. Chen, R. et al. Necrostatin- 1 protects $\mathrm{C} 2 \mathrm{C} 12$ myotubes from $\mathrm{CoCl}_{2}$-induced hypoxia. Int. J. Mol. Med. 41, 2565-2572 (2018).

35. Yoo, S. Y. et al. Neuregulin-1 protects neuronal cells against damage due to $\mathrm{CoCl}_{2}$-induced hypoxia by suppressing hypoxia-inducible factor-1a and P53 in SH-SY5Y cells. Int. Neurourol. J. 23, S111-S118 (2019).

36. Piret, J. P., Mottet, D., Raes, M. \& Michiels, C. $\mathrm{CoCl}_{2}$, a chemical inducer of hypoxiainducible factor-1, and hypoxia reduce apoptotic cell death in hepatoma cell line HepG2. Ann. N. Y Acad. Sci. 973, 443-447 (2002)

37. Grasselli, F., Basini, G., Bussolati, S. \& Bianco, F. Cobalt chloride, a hypoxiamimicking agent, modulates redox status and functional parameters of cultured swine granulosa cells. Reprod. Fertil. Dev. 17, 715-720 (2005).

38. Fang, Z., Luo, W. \& Luo, Y. Protective effect of a-mangostin against $\mathrm{CoCl}_{2}$-induced apoptosis by suppressing oxidative stress in $\mathrm{H} 9 \mathrm{C} 2$ rat cardiomyoblasts. Mol. Med. Rep. 17, 6697-6704 (2018).

39. Rana, N. K., Singh, P. \& Koch, B. $\mathrm{CoCl}(2)$ simulated hypoxia induce cell proliferation and alter the expression pattern of hypoxia associated genes involved in angiogenesis and apoptosis. Biol. Res. 52, 12 (2019).

40. Ke, Q. \& Costa, M. Hypoxia-inducible factor-1 (HIF-1). Mol. Pharmacol. 70, 1469-1480 (2006). 
41. Serocki, M. et al. miRNAs regulate the HIF switch during hypoxia: a novel therapeutic target. Angiogenesis 21, 183-202 (2018).

42. Huang, Y. et al. Hypoxia-preconditioned olfactory mucosa mesenchymal stem cells abolish cerebral ischemia/reperfusion-induced pyroptosis and apoptotic death of microglial cells by activating HIF-1a. Aging (Albany NY) 12, 10931-10950 (2020).

43. Fabian, M. R., Sonenberg, N. \& Filipowicz, W. Regulation of mRNA translation and stability by microRNAs. Annu. Rev. Biochem. 79, 351-379 (2010).

44. Mao, J. H. et al. miR-140-3p exhibits repressive functions on preosteoblast viability and differentiation by downregulating MCF2L in osteoporosis. Vitr. Cell Dev. Biol. Anim. 56, 49-58 (2020).

45. Liu, P. et al. miR-140-3p regulates the osteogenic differentiation ability of bone marrow mesenchymal stem cells by targeting spred2-mediated autophagy. Mol. Cell Biochem. 476, 4277-4285 (2021).

46. Karlsen, T. A., Jakobsen, R. B., Mikkelsen, T. S. \& Brinchmann, J. E. microRNA-140 targets RALA and regulates chondrogenic differentiation of human mesenchymal stem cells by translational enhancement of SOX9 and ACAN. Stem Cells Dev. 23, 290-304 (2014).

47. Feng, G. et al. SIRT1 was involved in TNF-a-promoted osteogenic differentiation of human DPSCs through Wnt/ $\beta$-catenin signal. Vitr. Cell Dev. Biol. Anim. 52, 1001-1011 (2016).

48. Sun, H. J. et al. A proteomic analysis during serial subculture and osteogenic differentiation of human mesenchymal stem cell. J. Orthop. Res. 24, 2059-2071 (2006).

49. Zhang, J. et al. Depletion of PRDM9 enhances proliferation, migration and chemotaxis potentials in human periodontal ligament stem cells. Connect Tissue Res. 61, 498-508 (2020).

50. Shen, W. C. et al. Methylation and PTEN activation in dental pulp mesenchymal stem cells promotes osteogenesis and reduces oncogenesis. Nat. Commun. 10, 2226 (2019).

51. Rodas-Junco, B. A., Canul-Chan, M., Rojas-Herrera, R. A., De-la-Peña, C. \& Nic-Can, G. I. Stem cells from dental pulp: what epigenetics can do with your tooth. Front. Physiol. 8, 999 (2017).
52. Deng, P., Chen, Q. M., Hong, C. \& Wang, C. Y. Histone methyltransferases and demethylases: regulators in balancing osteogenic and adipogenic differentiation of mesenchymal stem cells. Int. J. Oral. Sci. 7, 197-204 (2015).

53. Hui, $\mathrm{T}$. et al. EZH2, a potential regulator of dental pulp inflammation and regeneration. J. Endod. 40, 1132-1138 (2014).

54. Yi, Q. et al. Analysis of senescence-related differentiation potentials and gene expression profiles in human dental pulp stem cells. Cells Tissues Organs 203, 1-11 (2017).

55. Liu, H. et al. GREM1 inhibits osteogenic differentiation, senescence and BMP transcription of adipose-derived stem cells. Connect Tissue Res. 62, 325-336 (2021).

56. Han, X. et al. miR-4651 inhibits cell proliferation of gingival mesenchymal stem cells by inhibiting HMGA2 under nifedipine treatment. Int. J. Oral. Sci. 12, 10 (2020).

57. Zhang, C. et al. The histone demethylase KDM3B promotes osteo-/odontogenic differentiation, cell proliferation, and migration potential of stem cells from the apical Papilla. Stem Cells Int. 2020, 8881021 (2020).

(i) Open Access This article is licensed under a Creative Commons Attribution 4.0 International License, which permits use, sharing, adaptation, distribution and reproduction in any medium or format, as long as you give appropriate credit to the original author(s) and the source, provide a link to the Creative Commons license, and indicate if changes were made. The images or other third party material in this article are included in the article's Creative Commons license, unless indicated otherwise in a credit line to the material. If material is not included in the article's Creative Commons license and your intended use is not permitted by statutory regulation or exceeds the permitted use, you will need to obtain permission directly from the copyright holder. To view a copy of this license, visit http://creativecommons. org/licenses/by/4.0/.

(c) The Author(s) 2021 